\title{
Editorial
}

\section{Vaporizer overfilling}

Anaesthetic equipment has improved with regard to the ease of administration of anaesthetic agents and patient safety. Vaporizers are no exception. Agent-specific, concentration-calibrated, temperature-compensated vaporizers were devised to deliver direct reading, predictable concentrations of anaesthetic vapours delivered to the anaesthetic circuit without having to calculate vaporizer output and compensate for temperature changes during use. However, erroneous filling of agent-specific vaporizers with the wrong agent may occur quite frequently, especially in a large institution with a large anaesthesia staff and a high percentage of students, and lead to the unknowing delivery of markedly different concentrations than expected. ${ }^{1}$

The Keyed Filling Devices (KFD) presently in use "ensure that a vaporizer of an anaesthesia machine is filled only with the intended liquid anaesthetic" (CSA Standard Z168.4-M83). It is composed of a colour-coded bottle adaptor, keyed at both ends to fit only the bottle and the vaporizer filler receptacle for which it was devised. To fill a vaporizer, the keyed bottle adaptor is screwed on tightly - to ensure an airtight joint - to the matching bottle of anaesthetic agent. The keyed end of the adaptor is inserted fully into the vaporizer receptacle and tightened by a screw clamp to secure the alignment between the inner tubes of the adaptor and the holes in the receptacle. Finally, with the concentration dial in the "Off" position, the filler control is opened and the liquid in the bottle flows into the vaporizer through the filler tube, replacing air from the vaporizer which flows back through the air return tube. Used correctly, the system serves another important function, the prevention of overfilling, by two mechanisms. First, the intake of air to the bottle of anaesthetic agent is interrupted when filling has reached the maximum safe level of liquid in the vaporizing chamber. Second, by leaving the concentration dial in the "Off" position, the air cavity at the top of the vaporizing chamber is sealed, thereby preventing overfilling.

Unfortunately, the device is not perfect. Without the adaptor, it is impossible to fill the vaporizer. Defective

Chairman, Standards of Practice Committee, Canadian Anaesthetists' Society. bottle collars prevent fitting of the correct bottle to the appropriate filler, ${ }^{2}$ or allow fitting of the wrong bottle to the adaptor ${ }^{2,3}$ while not forming an airtight seal because of thread incompatibility. ${ }^{4}$ Oversized bottles may damage the threads of metal fillers or prevent an airtight seal between filler and bottle. ${ }^{5}$ If the holes in the adaptor and the receiver are not aligned properly, filling may be difficult. Filling is also slower than with the funnel type filling system.

Slow or difficult filling with the KFD have led individuals to adopt improper filling procedures to accelerate filling. Loosening the seal between the bottle and the adaptor to allow entry of room air into the bottle and turning the concentration dial to the "On" position were found to promote rapid filling. In this issue of the CJA, Sinclair and Van Bergen report that these procedural errors also led to the overfilling of all vaporizers in a Windsor hospital and, presumably, to a subsequent halothane overdose in a three-year-old child. ${ }^{6}$ Their letter stresses the double fault condition required for overfilling to occur, describes the effects of overfilling on vaporizer performance, and explains why overfilling is possible despite certification of the involved equipment to CSA Standard Z168.3-M84. They recommend that vaporizers with the KFD be equipped with an overfill drain.

In order to avoid the repetition of such a tragic accident, three other recommendations are in order. First, the use of agent-specific monitors could help detect abnormally high or low concentrations of anaesthetic vapours and alert the clinician to equipment malfunction. Second, the KFD should be improved to provide troublefree filling of vaporizers, thus avoiding the temptation to resort to improper procedures to facilitate or hasten filling. Third, and most important, users of these sophisticated pieces of medical equipment must read and follow instructions. Filling of vaporizers must not be delegated to a person without proper instruction and training. Overfilling of vaporizers is a well known hazard. All the appropriate recommendations to avoid overfilling are contained in standard textbooks ${ }^{8}$ and in the user instruction manuals accompanying the equipment. Of particular importance to prevent overfilling, the operator must not break the airtight junction between the bottle and the $\mathrm{KFD}$, and the concentration dial must be left in the "Off" position during filling. The liquid level in the sight glass 
must be monitored during and after filling to verify that the recommended maximum filling level has not been exceeded. A vaporizer that has been overfilled must be withdrawn from use.

Finally, it is the role of our Society and of the Journal to make certain that such critical information is available to clinicians on a timely basis. A reminder on the correct filling procedure has been published in the newsletter of the Canadian Anaesthetists' Society. The Journal invited a letter by the Bureau of Radiation and Medical Devices (Health Protection Branch, Health and Welfare Canada) on vaporizer overfilling. ${ }^{6}$ This editorial comment entreats clinicians, for the sake of patient safety, to follow instructions ... please.

\section{Remplissage excessif des vaporisateurs}

L'équipement anesthésique moderne tend à rendre plus facile l'administration des agents anesthésiques et à améliorer la sécurité des patients. Les vaporisateurs ne font pas exception à la règle. Pour ne plus avoir à calculer la concentration des vapeurs anesthésiques produites par le vaporisateur, et pour compenser les variations de cette concentration en fonction de la température, les vaporisateurs à lecture directe et à débit compensé pour la température ont été mis au point. Ils permettent l'administration fiable d'une concentration précise d'agent volatil dans le circuit anesthésique. Cependant, le remplissage accidentel du vaporisateur avec un agent autre que celui pour lequel il a été conçu survient encore relativement fréquemment, tout spécialement dans les grandes institutions où oeuvre un personnel anesthésique nombreux avec un haut pourcentage d'étudiants. De telles erreurs de remplissage peuvent mener à l'administration de concentrations d'agent volatil très différentes des concentrations prévues. ${ }^{1}$

Les dispositifs de remplissage clavetés pour vaporisateur anesthésique (DRC) sont présentement utilisés de façon à s'assurer qu'un vaporisateur anesthésique ne soit rempli qu'avec l'agent volatil qui lui est destiné (Norme Nationale CSA Z168.4-M83). Ce dispositif est composé d'un adaptateur de couleur spécifique, claveté à chaque extrémité pour ne s'ajuster qu'au contenant et au réceptacle du vaporisateur pour lequel il a été mis au point. Lorsqu'un vaporisateur doit être rempli, l'adaptateur claveté est vissé fermement - de façon à assurer un joint étanche - à la bouteille d'agent anesthésique approprié. L'extrémité clavetée de l'adaptateur est ensuite insérée jusqu'au fond du réceptacle du vaporisateur et maintenue en place par la vis de serrage de façon à assurer l'alignement des tubes internes de l'adaptateur avec les orifices du réceptacle. Finalement, en s'assurant que le cadran de concentration est à la position " $O$ ", le robinet de contrôle de remplissage du vaporisateur est ouvert, ce qui permet au liquide dans la bouteille de s'écouler dans le vaporisateur à travers le tube de remplissage, en échange de l'air contenu dans le vaporisateur qui s'échappera vers la bouteille à travers la tubulure de retour d'air.

Utilisé de façon correcte, ce dispositif remplit une autre fonction importante, la prévention du remplissage excessif et ce, par deux mécanismes. Premièrement, l'arrivée d'air dans la bouteille d'agent anesthésique est interrompue lorsque le remplissage a atteint le niveau maximum sécuritaire de liquide dans la chambre de vaporisation. Deuxièmement, lorsque le cadran de concentration est maintenu à la position " $\mathrm{O}$ ", la cavité aérique au sommet de la chambre de vaporisation est isolée, empêchant ainsi le sur-remplissage.

Malheureusement, le dispositif comporte des imperfections. Sans le dispositif, il est impossible de remplir un vaporisateur. L'installation sur une bouteille d'un collier défectueux empêchera de joindre la bouteille à l'adaptateur approprié, ${ }^{2}$ ou autorisera la mise en place du mauvais adaptateur sur une bouteille $e^{2,3}$ tout en empêchant la formation d'un joint étanche à cause de l'incompatibilité dans les pas de vis différents. ${ }^{4}$ Des bouteilles surdimensionnées peuvent endommager les pas de vis des adaptateurs métalliques ou prévenir la formation d'un joint étanche entre l'adaptateur et la bouteille. ${ }^{5} \mathrm{Si}$ les orifices de l'adaptateur et du réceptacle ne sont pas bien alignés, le remplissage peut être difficile. Le remplissage est généralement plus lent qu'avec les systèmes de type entonnoir.

Certaines personnes ont été tentées d'adopter des procédures de remplissage incorrectes de façon à accélérer le remplissage des vaporisateurs rendu difficile ou ralenti par le DRC. Ces personnes se sont aperçu qu'en dévissant légèrement l'adaptateur pour permettre l'entrée d'air ambiant dans la bouteille et qu'en ouvrant le cadran de concentration, il était possible d'accélérer de beaucoup le remplissage. Dans ce numéro du Journal Canadien d'Anesthésie, Sinclair et Van Bergen rapportent que de telles erreurs de procédure sont à l'origine du remplissage excessif de tous les vaporisateurs dans un hôpital de la région de Windsor. ${ }^{6} \mathrm{Ce}$ sur-remplissage est probablement responsable d'un surdosage d'halothane chez un enfant de trois ans. ${ }^{6}$ Leur lettre met l'emphase sur la double erreur de procédure nécessaire pour produire ce tropplein, décrit les effets d'un remplissage excessif sur le ren- 
dement du vaporisateur, et explique comment ce remplissage excessif a pu survenir malgré la conformité des vaporisateurs impliqués à la Norme Nationale CSA Z168.3-M84. Ils recommandent que les vaporisateurs munis du DRC soient équipés d'un drain prévenant le trop-plein.

Dans le but d'éviter la répétition d'incidents tragiques semblables, trois autres recommandations sont de mise. Premièrement, l'utilisation de moniteurs capables didentifier l'agent anesthésique administré, et de détecter des concentrations anormalement élévées ou diminuées? de vapeur, pourraient alerter le clinicien d'une anomalie de fonctionnement de l'équipement. Deuxièmement, le DRC devrait être amélioré de façon à assurer le remplissage facile et rapide des vaporisateurs, délivrant le clinicien de la tentation de recourir à des procédures incorrectes de remplissage. Troisièmement, les utilisateurs de ces équipements médicaux spécialisés doivent absolument lire et suivre les instructions. Le remplissage des vaporisateurs ne doit pas être délégué à une personne n'ayant pas reçu la formation appropriée. Le sur-remplissage des vaporisateurs est un danger bien connu. Toutes les recommandations appropriées dans le but d'éviter ce danger se retrouvent dans les manuels de base ${ }^{8}$ et dans les livrets d'instruction accompagnant l'équipement. Tout particulièrement, si l'on veut éviter de trop remplir un vaporisateur, il ne faut jamais briser le joint d'étanchéité entre la bouteille et l'adaptateur, et le cadran de concentration doit, absolument, sans faute, être laissé à la position " $O$ " durant le remplissage. Le ménisque liquidien dans la colonne de monitorage de niveau doit être surveillé pendant et après le remplissage de façon à s'assurer que le niveau maximal sécuritaire de liquide n'a pas été dépassé. Un vaporisateur qui a fait l'objet d'un trop-plein doit être retiré de la circulation sur le champ.

Finalement, notre Société et le Journal doivent s'assurer que ces informations vitales sont transmises aux cliniciens dans les meilleurs délais. Un rappel sur la procédure à suivre pour remplir un vaporisateur correctement sera publié prochainement dans la lettre de nouvelles de la Société Canadienne des Anesthésistes. Le Journal a invité la Direction Générale de la Protection de la Santé (Santé et Bien-Etre Social Canada) à nous fournir ses commentaires sur le remplissage excessif des vaporisateurs. ${ }^{6}$ Cet éditorial demande aux cliniciens, pour la sécurité de leurs patients, de bien vouloir suivre les instructions ... s'il vous plait.

\section{References}

1 Karis $J H$, Menzel $D B$. Inadvertent change of volatile anesthetics in anesthesia machines. Anesth Analg 1982; 61: 53-5.

2 George TM. Failure of keyed agent-specific devices (Letter). Anesthesiology 1984; 61: 228-9.
3 Riegle EV, Desertspring D. Failure of the agent-specific filling device (Letter). Anesthesiology 1990; 73: 353-4.

4 Ferstandig $L L$. Failure of the agent-specific filling device (Reply). Anesthesiology 1990; 73: 354.

5 Medical Devices Alert No 101. Leakage and breakage of anaesthetic bottles of Forane and possible damage to metal filling adaptors. Health Protection Branch, Ottawa, 1992.

6 Sinclair A, Van Bergen J. Vaporizer overfilling (Letter). Can J Anaesth 1992; 40: 77.

7 Slinger $P D$, Scott $W A C$, Kliffer $A P$. Intraoperative awareness due to malfunction of a Siemens $900 \mathrm{~B}$ ventilator. Can J Anaesth 1990; 37: 258-61.

8 Dorsch $J A$, Dorsch $S E$. Understanding Anesthesia Equipment. 2nd ed. Baltimore: Williams and Wilkins, 1984. 